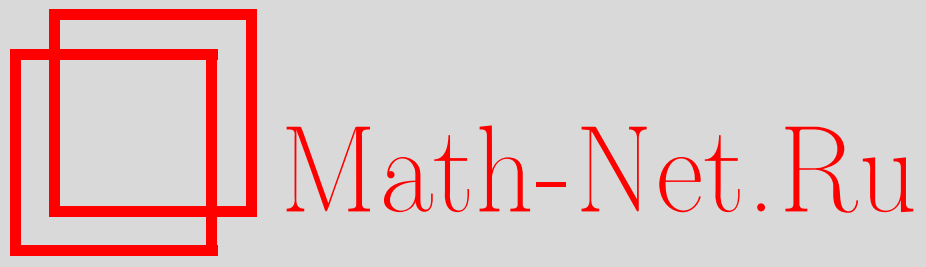

Ш. А. Аюпов, Описание вещественных алгебр фон Неймана с абелевой косоэрмитовой частью, Функи. анализ и его прил., 2002, том 36, выпуск 2, 75-77

DOI: https://doi.org/10.4213/faa193

Использование Общероссийского математического портала MathNet.Ru подразумевает, что вы прочитали и согласны с пользовательским соглашением

http://www . mathnet.ru/rus/agreement

Параметры загрузки:

IP: 54.162 .27 .143

26 апреля 2023 г., 17:15:40

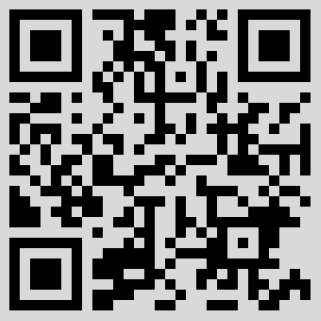




\title{
Описание вещественных алгебр фон Неймана с абелевой косоэрмитовой частью
}

\author{
(c) 2002. Ш. А. Аюпов
}

Рассмотрим *-алгебру $B(H)$ всех ограниченных линейных операторов в комплексном гильбертовом пространстве $H$. Напомним $[1,2]$, что слабо замкнутая вещественная *-подалгебра $R$ в $B(H)$ называется вещественной алгеброй фон Неймана, если она содержит единичный оператор $\mathbb{I}$ и $R \bigcap i R=\{0\}$. Имеется очень тесная связь между вещественными алгебрами фон Неймана и инволютивными (т. е. периода 2) *-антиавтоморфизмами (комплексных) алгебр фон Неймана (подробнее см. $[1,2]$ ).

Для вещественной алгебры фон Неймана $R$ множество $R_{s}=\left\{x \in R: x^{*}=x\right\}$ всех ее эрмитовых элементов является слабо замкнутой йордановой алгеброй самосопряженных операторов ( $J W$-алгеброй [3]) относительно симметризованного произведения $x \circ y=\frac{1}{2}(x y+y x)$. Совокупность $R_{k}=\left\{x \in R: x^{*}=-x\right\}$ всех косоэрмитовых элементов является алгеброй Ли относительно коммутатора $[x, y]$.

Из результатов работы [4] следует, что если вещественная алгебра фон Неймана $R$ абелева, то она изоморфна прямой сумме алгебр вида $L^{\infty}(\Omega, \mu, \mathbb{R})$ и $L^{\infty}(\Omega, \mu, \mathbb{C})$, т. е. алгебр существенно ограниченных измеримых вещественных или комплексных функций на пространстве с мерой $(\Omega, \mu)$. Этот результат был обобщен в работе [5], где было доказано, что если $R-$ вещественная алгебра фон Неймана с абелевой эрмитовой частью $R_{s}$, то, кроме указанных выше, могут еще быть прямые слагаемые вида $L^{\infty}(\Omega, \mu, \mathbb{Q})$, где $\mathbb{Q}$ - тело кватернионов.

$\mathrm{B}$ настоящей заметке мы опишем вещественные алгебры фон Неймана $R, \mathrm{y}$ которых косоэрмитова часть $R_{k}$ является абелевой алгеброй Ли, т. е. $[x, y]=0$ для всех $x, y \in R_{k}$. А именно, будет доказан следующий результат:

Теорема. Пусть $R$ - вещественная алгебра фон Неймана, у которой косоэрмитова часть $R_{k}$ абелева. Тогда $R$ изоморфна прямой сумме алгебр следующего вида:

(i) $L^{\infty}(\Omega, \mu, \mathbb{R})$;

(ii) $L^{\infty}(\Omega, \mu, \mathbb{C})$;

(iii) $L^{\infty}\left(\Omega, \mu, M_{2}(\mathbb{R})\right)=L^{\infty}(\Omega, \mu, \mathbb{R}) \otimes M_{2}(\mathbb{R})$, где $M_{2}(\mathbb{R})$ - алгебра вещественных $2 \times 2$-матрии.

Прежде чем приступить к доказательству теоремы, приведем несколько вспомогательных лемм.

Лемма 1 (Putnam [6]). Пусть $a, x \in B(H)$, причем $a-$ нормальный оператор, m. е. $a^{*} a=a a^{*}$. Тогда если $[a,[a, x]]=0$, то $[a, x]=0$.

ЛЕмма 2. В условиях теоремы произведение ху для любых $x, y \in R_{k}$ является центральным элементом в $R$, т.е. коммутирует со всеми элементами в $R$.

ДокАЗАТЕльство. Так как $R_{k}$ абелева, то $(x y)^{*}=x y \in R_{s}$, причем $x y$ коммутирует со всеми элементами из $R_{k}$. Далее, так как $x y \in R_{s}$, то для любого $a \in R_{s}$ коммутатор $[a, x y]$ лежит в $R_{k}$ и поэтому коммутирует с $x$ и с $y$, а значит, и с элементом $x y$, т. е. $[[a, x y], x y]=0$. Поскольку элемент $x y \in R_{s}$ нормален, то, 
согласно лемме $1,[a, x y]=0$ для любого $a \in R_{s}$. Следовательно, $x y$ коммутирует со всеми элементами из $R=R_{s}+R_{k}$.

Лемма 3. В условиях теоремь, если $J W$-алгебра $R_{s}$ абелева, то вещественная алгебра фон Неймана $R$ коммутативна.

ДОКАЗАТЕЛЬСТВО. По условию $[x, y]=0$ для $x, y \in R_{k}$ и $[a, b]=0$ для всех $a, b \in R_{s}$. Поэтому достаточно показать, что $[a, x]=0$ для $a \in R_{s}, x \in R_{k}$. Но $[a, x]^{*}=[a, x] \in R_{s}$ и потому $[a,[a, x]]=0$. Так как $a^{*}=a$, то из леммы 1 следует, что $[a, x]=0$.

Лемма 4. В условиях теоремы любое семейство попарно ортогональных и эквивалентных проекторов в $J W$-алгебре $R_{s}$ содержит не более двух элементов.

ДокАЗАТЕЛЬСтво. Допустим противное, т.е. существуют три попарно ортогональных и попарно эквивалентных проектора $e=e_{1}, e_{2}, e_{3}$. В силу [3, Prop. 10] они связаны с помощью симметрии, т.е. существуют симметрии $s_{2}, s_{3} \in R_{s}$ $\left(s_{2}^{2}=s_{3}^{2}=\mathbb{I}\right)$, такие, что $e_{2}=s_{2} e s_{2}, e_{3}=s_{3} e s_{3}$. Рассмотрим элемент $x=$ $s_{2} e s_{3}-s_{3} e s_{2} \in R_{k}$. Учитывая ортогональность проекторов $e, e_{2}, e_{3}$, легко подсчитать, что $x^{2}=-e_{2}-e_{3}$. В силу леммы 2 элемент $x^{2}$ должен быть центральным в $R$. С другой стороны, рассмотрим элемент $a=e s_{2}+s_{2} e \in R_{s}$. Снова учитывая ортогональность проекторов $e, e_{2}, e_{3}$ и связывающие их соотношения с симметриями, нетрудно подсчитать, что

$$
\left[a, x^{2}\right]=-\left[e s_{2}+s_{2} e, e_{2}+e_{3}\right]=s_{2} e-e s_{2} .
$$

Следовательно,

$$
\left[a, x^{2}\right]^{2}=\left(s_{2} e-e s_{2}\right)^{2}=-e_{2}-e \neq 0,
$$

что противоречит центральности элемента $x^{2}$, т.е. $\left[a, x^{2}\right]=0$ для всех $a \in R$. Противоречие.

ДОКАЗАТЕЛЬСТВО тЕОРЕМЫ. В силу леммы 4 в $J W$-алгебре $R_{s}$ существует центральный проектор $z$, такой, что $z R_{s}$ имеет тип $\mathrm{I}_{1}$, т. е. является абелевой $J W$-алгеброй; $(\mathbb{I}-z) R_{s}$ имеет тип $\mathrm{I}_{2}$. Элемент $z$, центральный в $R_{s}$, автоматически является центральным в $R$. В самом деле, для $x \in R_{k}$ коммутатор $[z, x]$ лежит в $R_{s}$ и потому $[z,[z, x]]=0$, а согласно лемме $1,[z, x]=0$, т. е. $z$ коммутирует с любым элементом из $R_{k}$. Таким образом, $R=z R \oplus(\mathbb{I}-z) R$, где вещественная алгебра фон Неймана $z R$ имеет абелеву эрмитову часть $z R_{s}$ и абелеву косоэрмитову часть $(z R)_{k}=z R_{k}$. По лемме 3 вещественная алгебра фон Неймана $z R$ коммутативна и в силу [4], как уже упоминалось, является прямой суммой алгебр вида (i) и (ii). Осталось рассмотреть случай, когда $R_{s}$ есть $J W$-алгебра muna $\mathrm{I}_{2}$.

Так как $a_{1} \cdots a_{n}+a_{n} a_{n-1} \cdots a_{1} \in R_{s}$ для любых $a_{1}, \ldots, a_{n} \in R_{s}$, то $J W$-алгебра $R_{s}$ является обратимой $J W$-алгеброй типа $\mathrm{I}_{2}$. Известно [7], что любая $J W$-алгебра типа $\mathrm{I}_{2}$ разлагается в прямую сумму $J W$-алгебр

$$
\sum_{m=3}^{\infty} L^{\infty}\left(\Omega_{m}, \mu_{m}, V_{m}\right),
$$

где $\Omega_{m}$ - локально компактное хаусдорфово пространство, $\mu_{m}-$ мера Радона на $\Omega_{m}$ и $V_{m}-$ спин-фактор (т. е. $J W$-фактор типа $\mathrm{I}_{2}$ ) размерности $m \geqslant 3$. С другой стороны, если исходная $J W$-алгебра обратима, то обратимы и спинфакторы $V_{m}$, что возможно только при $m=3,4$ или 6 (см. [8]), т.е. когда 
$V_{3} \cong M_{2}(\mathbb{R})_{s}, V_{4} \cong M_{2}(\mathbb{C})_{s}$ либо $V_{6} \cong M_{2}(\mathbb{Q})_{s}$. Известно [9, Theorem 3], что любой вещественный фактор $M$, кроме $\mathbb{Q}$, обладает свойством $M_{k}=\left[M_{s}, M_{s}\right]$, т. е. любой косоэрмитов элемент есть конечная сумма коммутаторов эрмитовых элементов. В частности, косоэрмитовы матрицы из $M_{2}(\mathbb{F})$ алгебраически порождаются $M_{2}(\mathbb{F})_{s}$ (где $\mathbb{F}=\mathbb{R}, \mathbb{C}$ или $\left.\mathbb{Q}\right)$. Поэтому, если обратимая $J W$-алгебра $R_{s}$ содержит прямые слагаемые вида $L^{\infty}\left(\Omega, \mu, M_{2}(\mathbb{F})_{s}\right)$, то $R$ содержит слагаемое вида $L^{\infty}\left(\Omega, \mu, M_{2}(\mathbb{F})\right)$. Но в случае $\mathbb{F}=\mathbb{C}$ или $\mathbb{Q}$ алгебра $M_{2}(\mathbb{F})$ содержит косоэрмитовы матрицы, которые не коммутируют, что противоречит условию теоремы. Поэтому $R_{s}=L^{\infty}\left(\Omega, \mu, M_{2}(\mathbb{R})_{s}\right)$.

Как мы отметили выше, $R$ тогда содержит алгебру $R^{0}=L^{\infty}\left(\Omega, \mu, M_{2}(\mathbb{R})\right)$. Покажем, что на самом деле $R=R^{0}$. Итак, имеем $R_{s}=R_{s}^{0}$. Пусть $x \in R_{k}$. Рассмотрим произвольный обратимый косоэрмитов элемент $u$ из $R_{k}^{0}$, например, функцию на $\Omega$, тождественно равную матрице $\left(\begin{array}{cc}0 & -1 \\ 1 & 0\end{array}\right)$ из $M_{2}(\mathbb{R})$. Тогда $x u$ является эрмитовым элементом в $R_{s}=R_{s}^{0}$, т. е. $x=(x u) u^{-1} \in R_{s}^{0} R_{k}^{0} \subset R^{0}$. Таким образом, $R_{k}=R_{k}^{0}$, т.е. $R=R_{s}+R_{k}=R^{0}$. Итак, мы доказали, что если $R_{s}$ имеет тип $\mathrm{I}_{2}$, то $R=L^{\infty}\left(\Omega, \mu, M_{2}(\mathbb{R})\right)$, т. е. имеет место случай (iii). Теорема доказана.

\section{ЛИТЕРАТУРА}

1. Ayupov Sh. A., Rakhimov A. A., Usmanov Sh. M. Jordan, Real and Lie Structures in Operator Algebras. Kluwer Acad. Publ., Dordrecht, 1997. 2. Stormer E. Pacific J. Math., 21, No. 2, 349-370 (1967). 3. Topping D. Mem. Amer. Math. Soc., 53 (1965). 4. Li Minli, Li-Bingren. Acta Math. Sinica, 14, No. 1, 85-90 (1998). 5. Аюпов Ш. А., Рахимов А. А., Абдуваитов А. Х. Докл. АН РУз, №5 (2001). 6. Putnam C. R. Amer. J. Math. 73, 357-362 (1950). 7. Stacey P. J. Quart J. Math. Oxford, 33, No. 129, 115-127 (1982). 8. Robertson A. G. Quart. J. Math. Oxford, 34, No. 133, 87-96 (1983). 9. Ayupov Sh. A., Azamov N. A. Comm. Algebra, 24, No. 4, 1501-1520 (1996).

Институт математики АН Республики Узбекистан e-mails: e_ayupov@hotmail.com, ayupov@im.tashkent.su

Поступило в редакцию 7 мая 2001 г.

УДК 517.986

\section{О точках ветвления спектра алгебраического расширения равномерной алгебры}

(C) 2002. Б. Т. БАТИКян

Пусть $A[t]$ - алгебра всех полиномов над полупростой коммутативной банаховой алгеброй $A$ с единицей над полем $\mathbb{C}$ комплексных чисел. Совокупность унитарных полиномов обозначается через $A_{U}[t]$. Пусть $f \in A_{U}[t]$,

$$
f=t^{n}+a_{1} t^{n-1}+\cdots+a_{n}, \quad \operatorname{deg} f=n>1 .
$$

Обозначим через $B$ алгебраическое расширение алгебры $A$, порожденное полиномом $f$. Иначе говоря, $B=A[t] /(f)$, где $(f)$ - главный идеал в $A[t]$, порожденный $f$. 